\title{
A MECHANISM FOR ASTEROIDS TO AVOID CLOSE APPROACH AND COLLISION TO PLANETS
}

\author{
YOSHIHIDE KOZAI \\ Gunma Astronomical Observatory \\ Ootomo Branch Office of Gunma Prefecture, 1-18-7 Ootomo, Maebashi 371, Japan \\ e-mail:kozai@yso.mtk.nao.ac.jp
}

\begin{abstract}
.
In this paper it is explained how asteroids with large aphelion distances avoid very close approach to Jupiter. Namely, since for asteroids with resonant mean motions the critical arguments librate as it has been known for many years, and for those with non-resonant mean motions the eccentricities change with the argument of perihelion so the distance to Jupiter cannot be very small.

Also some behaviours of Kuiper-belt asteroids are studied by a similar way and it is found that the libration region for the argument of perihelion appears only when the eccentricity is very large. Therefore, even though the eccentricity is very large it is possible for any Kuiper-belt asteroid to avoid very close approach to any of the planets.
\end{abstract}

\section{Asteroids with large aphelion distances}

It is known that all asteroids with semi-major axes between 3.9 and $5.3 \mathrm{AU}$ have mean motions of $1: 1,7: 6,4: 3$ and $3: 2$ resonances as far as the numbered asteroids are concerned. However, as the critical arguments are librating around $\pm \pi / 3$ for Trojan asteroids and around 0 for the others except for those of Hilda group of small eccentricity, they do not approach Jupiter very closely. It is also true for 2:1 and other resonance cases.

There are a few asteroids, for which the aphelion distances are large. Namely, for some of them they are larger than $5 \mathrm{AU}$, even though their semi-major axes are less than $3.7 \mathrm{AU}$, that is, their mean motions are non-resonant. In Table 1 non-resonant asteroids with 5 largest aphelion distances are listed with necessary data.

In the Table the maximum and minimum values of the eccentricities and the inclinations are computed by adopting a simple model (Kozai, 1962, 1979). Namely, it is assumed that all the disturbing planets are moving along circular orbits on the same plane and the disturbing function is averaged with respect to the mean anomaly of the asteroid and the mean longitudes of the disturbing planets.

In Table 2 similar data for resonant asteroids with 10 largest aphelion distances are given. The mean motions of the asteroids in the Table are in $7: 6(a=4.7 \mathrm{AU}), 4: 3(4.2 \mathrm{AU}), 3: 2(4.0 \mathrm{AU})$ and

TABLE 1. Data for non-resonant asteroids with large aphelion distance

\begin{tabular}{|c|cc|c|c|cc|cc|cc|cc|}
\hline No. & $Q_{M}$ & $Q$ & $a$ & $r_{90}$ & $r_{1}$ & $r_{2}$ & $q_{M}$ & $Q_{m}$ & $i_{m}$ & $i_{M}$ & $e_{m}$ & $e_{M}$ \\
\hline 6130 & 4.82 & 4.59 & 2.99 & 1.87 & 1.39 & 4.55 & 1.40 & 4.58 & 10. & 23 & 0.53 & 0.61 \\
5324 & 4.88 & 4.78 & 2.96 & 1.71 & 1.23 & 3.66 & 1.42 & 4.51 & 13. & 30. & 0.52 & 0.65 \\
7604 & 4.91 & 4.90 & 3.12 & 2.09 & 1.97 & 2.24 & 2.07 & 4.16 & 19. & 35 & 0.34 & 0.57 \\
1373 & 5.30 & 4.51 & 3.40 & 2.34 & 2.86 & 3.23 & 3.05 & 3.05 & 29. & 40. & 0.32 & 0.56 \\
5164 & 5.54 & 5.49 & 3.66 & 2.69 & 2.31 & 3.36 & 2.75 & 4.57 & 17. & 32. & 0.25 & 0.51 \\
\hline
\end{tabular}


TABLE 2. Data for resonant asteroids with large aphelion distances

\begin{tabular}{|r|rr|r|r|rr|rr|rr|rr|}
\hline No. & $Q_{M}$ & $Q$ & $a$ & $r_{90}$ & $r_{1}$ & $r_{2}$ & $q_{M}$ & $Q_{m}$ & $i_{m}$ & $i_{M}$ & $e_{m}$ & $e_{M}$ \\
\hline 2959 & 5.02 & 5.03 & 3.94 & 3.64 & 3.48 & 3.80 & 2.93 & 4.95 & 4. & 7. & 0.26 & 0.28 \\
1877 & 5.03 & 4.78 & 3.94 & 3.65 & 3.30 & 4.39 & 3.33 & 4.56 & 15. & 20. & 0.16 & 0.27 \\
2483 & 5.11 & 5.06 & 3.97 & 3.65 & 2.89 & 5.06 & 2.89 & 5.06 & 2. & 6. & 0.27 & 0.29 \\
4446 & 5.11 & 5.10 & 3.98 & 3.65 & 3.37 & 4.00 & 3.05 & 4.91 & 7. & 12. & 0.23 & 0.28 \\
1941 & 5.11 & 5.10 & 3.99 & 3.68 & 3.03 & 4.70 & 2.92 & 5.07 & 2. & 5. & 0.27 & 0.28 \\
& & & & & & & & & & & & \\
5661 & 5.18 & 4.90 & 3.95 & 3.57 & 3.01 & 4.90 & 3.01 & 4.90 & 8. & 14. & 0.24 & 0.31 \\
1922 & 5.41 & 4.80 & 3.25 & 1.82 & 1.78 & 4.28 & 2.08 & 4.43 & 18. & 40. & 0.36 & 0.66 \\
5370 & 5.60 & 5.46 & 3.35 & 1.83 & 1.27 & 4.82 & 1.55 & 5.14 & 7. & 30. & 0.54 & 0.67 \\
6144 & 6.40 & 6.40 & 4.72 & 4.11 & 4.05 & 4.17 & 3.01 & 6.42 & 5. & 4. & 0.36 & 0.36 \\
3552 & 7.58 & 7.26 & 4.23 & 1.58 & 1.35 & 4.50 & 2.64 & 2.64 & 25. & 46. & 0.61 & 0.79 \\
\hline
\end{tabular}

2:1(3.3AU) resonances. In fact there are many high eccentricity asteroids in mean motion resonance regions except for the Hilda group.

\section{Secular Variations}

When Delaunay variables ( $\mu$ being the product of the constant of gravitation and the solar mass),

$$
\begin{aligned}
& L=\sqrt{\mu a}, \quad G=L \sqrt{1-e^{2}}, \quad H=G \cos i, \\
& l=\text { mean anomaly, } g=\varpi-\Omega, \quad h=\Omega \text {, }
\end{aligned}
$$

are introduced, they satisfy the canonical equations of three degrees of freedom with the Hamiltonian, $F$,

$$
F=\frac{\mu^{2}}{2 L^{2}}+\sum m^{\prime} / \Delta
$$

where $\Delta$ is the mutual distance with one of the disturbing planets and $m^{\prime}$ is its mass as the fraction of the solar mass.

Because of the assumption, the Hamiltonian is symmetric around the $z$-axis after the averaging with respect to the fast moving angular elements, the mean anomaly of the asteroid, $l$, and the mean longitudes of the disturbing planets, $\lambda$. Then, $h$, the longitude of the ascending node, does not appear in the Hamiltonian, and therefore, $H$, the $z$-component of the angular momentum, becomes constant. Since $l$ has been eliminated, $L$ is constant. Then the equations are reduced to a system of one degree of freedom with the integral $F=$ constant.

Then with the two parameters, $L$ and $H, G$ can be expressed as periodic functions of $g$, the argument of perihelion, in fact of $2 g$. In practice instead of $L, H$ and $G$,

$$
a, \quad \Theta=\sqrt{1-e^{2}} \cos i, \quad X=\sqrt{1-e^{2}},
$$

are used and the Hamiltonian is replaced by,

$$
F^{*}=\frac{1}{(2 \pi)^{2}} \sum \int_{0}^{2 \pi} \int_{0}^{2 \pi} \frac{m^{\prime}}{\Delta} d l d \lambda^{\prime} .
$$

For resonant asteroids the averaging is made under the assumption that the critical argument is fixed at 0 . That is in Table 2 , it is assumed that the critical argument for any asteroid is fixed.

For given values of $a$ and $\Theta$ the values of $F^{*}$ are computed for various sets of $X$ and $2 q$, and curves of equal $F^{*}$ values --. along which $X$ as well as $e$ and $i$ vary as functions of $2 g \cdots$ can be drawn. In fact, $X$ can take values between 1 and $\Theta$ which correspond, respectively, to $e=0$ and $i=\arccos \Theta$ and to $e=\sqrt{1-\Theta^{2}}$ and $i=0$. When $\Theta$ is nearly equal to 1 , any curve of equal $F^{*}$ value is a nearly horizontal line, namely, parallel to the $2 g$-axis. However, when $\Theta$ is well below 1 , the curves are no longer horizontal lines, and they show that the eccentricity is a minimum at $2 g=0$ and $2 \pi$, and a maximum at $2 g=\pi$ and vice versa for the inclination. 
By drawing such diagrams the variations of the two elements are traced. It is known that if the value of $\Theta$ is less than 0.8 a libration region appears, in which the argument of perihelion librates around $2 g=\pi$, and the eccentricity and the inclination take their maximum and minimum values at $2 g=\pi$.

In the Tables the first column gives the assigned number, the second and the third columns are, respectively, the maximum and the present aphelion distances and the fourth column expresses the semi-major axis. The fifth column, $r_{90}$, is the heliocentric distance of the ascending and the descending nodes of the orbital plane with respect to the reference plane when the eccentricity takes the maximum value, that is, at $2 g=\pi$, and this distance is computed as $a\left(1-e^{2}\right)$.

The sixth and seventh columns, $r_{1}$ and $r_{2}$, are the heliocentric distances of the ascending and the descending nodes computed by the present values of the eccentricity and the argument of perihelion. Then $q_{M}$ and $Q_{m}$ are the perihelion and aphelion distances for the minimum value of the eccentricity which corresponds to the case of $2 g=0$. And finally the maximum and minimum values of the inclinations and the eccentricities are given.

For the asteroids (1373) and (3552) the arguments of perihelion do not make complete revolutions but librate around $\pi / 2$ or $3 \pi / 2$ and the maximum and the minimum of the two elements occur there. Therefore, under $q_{M}$ and $Q_{m}$ the values of $a\left(1-e_{m}^{2}\right)$, the heliocentric distances of the nodes, where $2 l=\pi$, are given.

Table 1 shows that although the maximum and the present aphelion distances are large for the 5 asteroids, the heliocentric distances of the asteroids when they cross the reference plane, the orbital plane of the disturbing planets, are not so large as to approach Jupiter very closely. Still the largest heliocentric distances on the reference plane are 4.6 AU for some of the asteroids.

On the other hand, in Table 2 the heliocentric distances when the asteroids cross the reference plane are not necessarily small. In fact for (6144) the aphelion distance is as large as 6.4 AU and the inclination is small. However, since the critical arguments are librating, they do not approach Jupiter very closely.

In fact it is clear that the mechanism to avoid very close approach works for large eccentricity asteroids. And one can say that more eccentric asteroids are more stable in the sense that they do not approach Jupiter closely both for non-resonant and resonant cases.

\section{Kuiper-belt Asteroids}

It is already known that for asteroids of the Kuiper belt the eccentricities and the inclinations change as functions of the arguments of perihelion (Morbidelli, Thomas and Moons, 1995). However, they do not change so much as those for main-belt asteroids with similar values of $\Theta$ except for regions corresponding to the eccentricities such as $0.25,0.52,0.76$ and 0.87 , for which the perihelion distances are nearly equal to the semi-major axes of Neptune, Uranus, Saturn and Jupiter, respectively. In fact the value of $F^{*}$ takes its maximum near one of such eccentricities and $2 g=0$.

Generally the value of $F^{*}$ increases gradually as the value of $X=\sqrt{1-e^{2}}$ decreases and there is no libration region except for cases of small values of $\Theta$. However, for a case of resonant mean motion like the Pluto-Neptune case the value of $F^{*}$ is decreased as the eccentricity is increased if the critical argument is librating around $\pi$. Therefore, a libration region appears in the diagram, and it is known that the argument of perihelion for Pluto is librating.

If the value of $\Theta$ is very small, libration regions appear as it is shown for the case of $a=40$ $\mathrm{AU}$ and $\Theta=0.20$ in Figure 1. Under the horizontal axis the value of $2 g$ is plotted; on the left-hand side of the vertical axis the value of $X=\sqrt{1-e^{2}}$ and on the right-hand side the values of the eccentricity and the inclination are given.

If for any asteroid the eccentricity and the argument of perihelion are in one of the libration regions near $2 g=\pi$, as its perihelion is very far from the reference plane it never approaches any planet even though the eccentricity is very large.

In any case the mechanism discussed here plays an important role for highly eccentric orbit asteroids to avoid very close approach to major planets.

Finally it is remarked that since the eccentricities and the mutual inclinations of the disturbing planets are neglected any secular resonance is not taken into account in this paper. 


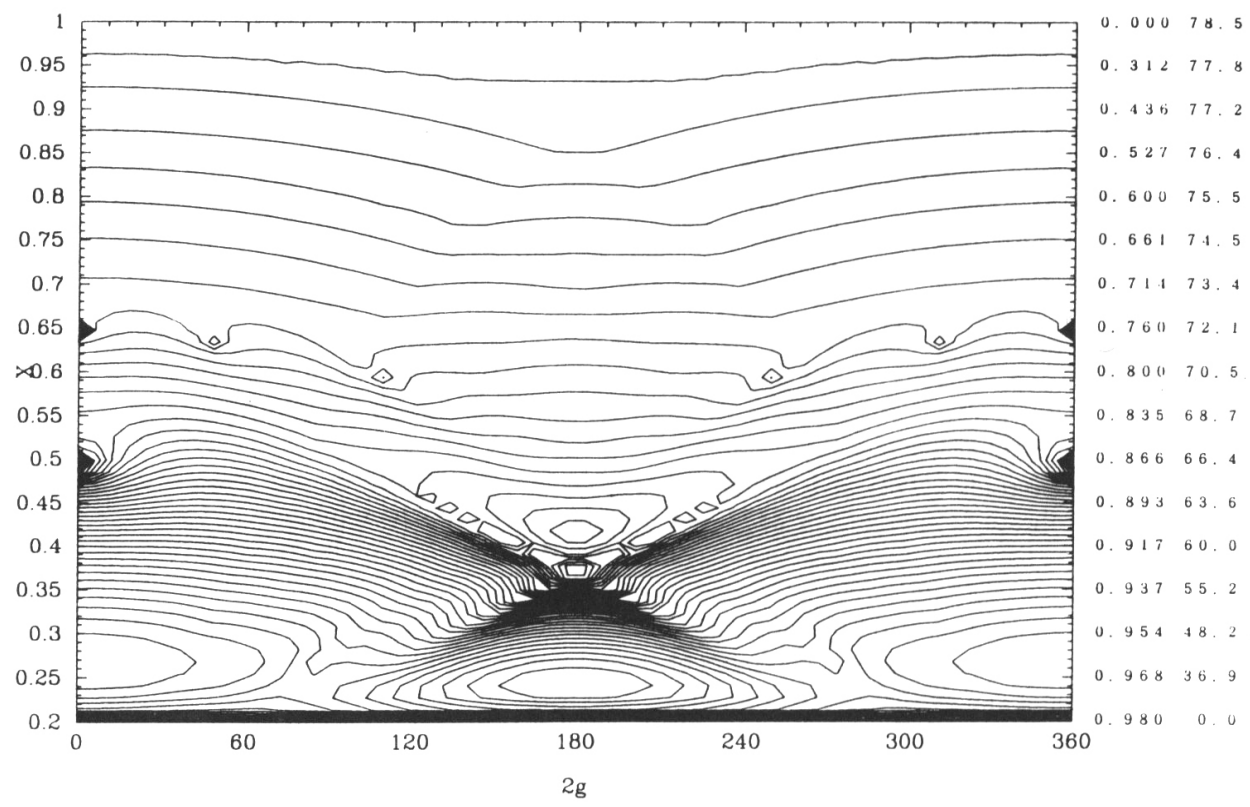

Figure 1. Diagrams for $a=40 \mathrm{AU}$ and $\Theta=0.20$.

\section{References}

Kozai, Y. 1962. Astron. J., 67, 591-198.

Kozai, Y. 1979. In Dynamics of the Solar System, ed. R.L. Duncombe, 231-237.

Morbidelli, A., Thomas, F. and Moons, M. 1995. Icarus, 118, 322-340. 